\title{
Módulo híbrido de membranas de microfiltração E CARVÃo ATIVAdo COM NANOPARTÍCULAS METÁLICAS
}

\author{
Natalia Ueda Yamaguchi ${ }^{1 *}$, Andressa Jenifer Rubio ${ }^{1}$, Luciana C. S. H. Rezende ${ }^{1}$, Rosangela Bergamasco ${ }^{1}$ \\ 1 Centro Universitário de Maringá, Av. Colombo, 5790 - Bloco D-90 - CEP 87020-900 - Maringá - PR \\ *Autor para correspondência: nataliaueda@hotmail.com
}

Recebido em 13 de agosto de 2019. Aceito em 14 de dezembro de 2019. Publicado em 27 de dezembro de 2019.

Resumo - Esta pesquisa contribuiu para o desenvolvimento de módulo híbrido gravitacional de membrana de microfiltração comercial de acetato de celulose e um material bactericida, carvão ativado granular impregnado com $0,5 \%$ de prata e $0,5 \%$ de cobre $(\mathrm{CAG} / \mathrm{AgCu})$. Os materiais adsorventes foram caracterizados por técnicas de adsorção de nitrogênio, microscopia eletrônica de varredura (MEV), espectrometria de energia dispersiva (EDX), microscopia eletrônica de transmissão (MET) e difração de raios-X (DRX). A eficiência do módulo híbrido foi verificada por meio da avaliação do (i) fluxo permeado, (ii) $\mathrm{pH}$, (iii) turbidez, (iv) remoção de cloro livre e (v) eficiência bactericida. O filtro apresentou maiores fluxos iniciais de permeado, não alterou o pH, não aumentou significativamente a turbidez, e apresentou remoções de cloro e Escherichia coli superiores a 90\% e 3,7 log respectivamente, revelando seu potencial na tecnologia de filtros para tratamento de água.

PaLAVRas-CHaVE: Cobre; filtração; impregnação; membrana; prata.

\section{HYBRID MODULE OF MEMBRANE MICROFILTRATION AND ACTIVATED CARBON WITH METAL NANOPARTICLES}

ABstract - This research has contributed to the development of a gravitational hybrid membrane module for commercial cellulose acetate microfiltration membrane and a bactericidal material, granular activated carbon impregnated with $0.5 \%$ silver and $0.5 \%$ copper $(\mathrm{CAG} / \mathrm{AgCu})$. The adsorbent materials were characterized by nitrogen adsorption techniques, scanning electron microscopy (SEM), dispersive energy spectrometry (EDX), transmission electron microscopy (MET) and X-ray diffraction (XRD). The efficiency of the hybrid module was verified by evaluating the (i) permeate flux, (ii) pH, (iii) turbidity, (iv) free chlorine removal and (v) bactericidal efficiency. The filtration module showed higher initial permeate fluxes, did not change the $\mathrm{pH}$, did not significantly increase turbidity and presented chlorine and Escherichia coli removal greater than $90 \%$ and $3.7 \mathrm{log}$, respectively. Thus, the studied module revealed its potential in water treatment filter technology.

KEYwORDs: copper, filtration, impregnation, membrane, silver.

\section{MÓDULO HÍBRIDO DE MEMBRANA DE MICROFILTRACIÓN Y CARBÓN ACTIVADO CON NANOPARTÍCULAS METÁLICAS}

Resumen - Esta investigación contribuyó al desarrollo de un módulo híbrido gravitacional de membrana de microfiltración comercial de acetato de celulosa y un material bactericida, carbón activado granular impregnado con $0.5 \%$ de plata y $0,5 \%$ de cobre $(\mathrm{GAC} / \mathrm{AgCu})$. Los materiales adsorbentes se caracterizaron por técnicas de adsorción de nitrógeno, microscopía electrónica de barrido (MEV), espectrometría de energía dispersiva (EDX), microscopía electrónica de transmisión (MET) y difracción de rayos-X (DRX). La eficiencia del módulo híbrido se verificó mediante la evaluación del (i) flujo de permeado, (ii) $\mathrm{pH}$, (iii) turbidez, (iv) eliminación de cloro libre y (v) eficiencia bactericida. El filtro presentó flujos de permeado iniciales más altos, no cambió el pH, no aumentó significativamente la turbidez y presentó una eliminación de cloro y Escherichia coli superiores al 90\% y 3.7 log, respectivamente. Por lo tanto, el módulo estudiado reveló su potencial en la tecnología de filtración aplicable al tratamiento de agua. 
Palabras clave: cobre; filtración; impregnación; membrana; plata.

\section{INTRODUÇÃO}

A qualidade da água é essencial para uma boa saúde e um fator importante na redução da mortalidade causada por doenças transmitidas pela água. Um dos fatores mais importantes para melhorar e proteger a saúde humana é o acesso à água potável. Milhões de pessoas morrem todos os anos devido à falta de acesso a água potável segura, livre de microorganismos patogênicos, e uma grande porcentagem deles está em países em desenvolvimento (Montgomery \& Elimelech 2007).

Em países em desenvolvimento o tratamento centralizado de água potável pode ser deficiente em relação à qualidade da água. A contaminação de água canalizada pode ocorrer em algum momento ao longo da distribuição da água ao consumidor final. Problemas como quedas de pressão no sistema de distribuição, avarias na tubulação, e deficiências na manutenção de estações de tratamento, precariedade na assepsia das caixas d'água podem ser apontados como fatores de risco de contaminação no processo de distribuição (Brick et al. 2004, Peter-Varbanets et al. 2009).

Um dos principais fatores de riscos relacionados ao consumo de água contaminada, estão associados à contaminação microbiológica. Isto ocorre, pois a água é um potencial veiculador de doenças, e pode atuar como portador passivo de patógenos infecciosos, principalmente relacionados à contaminação fecal (Peter-Varbanets et al. 2009). Devido às complexidades associadas à tentativa de detectar todos os possíveis patógenos nas fontes de água, indicadores, como bactérias Escherichia coli têm sido amplamente utilizadas para avaliar a qualidade microbiológica da água natural e da água para consumo humano (APHA et al. 2012).

Filtros domésticos simples têm sido utilizados para assegurar a qualidade da água quando o sistema centralizado não é eficiente no seu tratamento. Estes filtros atuam como um pós-tratamento para que o consumidor obtenha água com a qualidade adequada (Peter-Varbanets, et al., 2009). Tecnologias não convencionais de tratamento, como por exemplo: (a) radiação ultravioleta, (b) ozônio, (c) membranas, (d) filtração em carvão ativado, (e) zeólitas e (e) resinas de troca iônica, podem ser aplicadas aos filtros domésticos devido à alta eficácia destas tecnologias para remoção de diversos tipos de contaminantes (Silva et al. 2012).

Dentre as tecnologias disponíveis, o carvão ativado é um material carbonoso altamente poroso, amplamente utilizado no tratamento da água para remover poluentes orgânicos e inorgânicos devido às suas propriedades físicas e químicas ajustáveis, como porosidade controlável e altamente desenvolvida, grande área superficial, alta reatividade superficial e modificável superfície (Arakawa, Shimabuku-Biadola, Fernandes Silva, \& Bergamasco, 2019). Porém, apesar de sua área específica elevada, o carvão ativado não apresenta elevada eficiência bactericida. A modificação de sua superfície tem se mostrado uma abordagem eficaz para aumentar o poder bactericida do carvão ativado (Bhatnagar et al. 2013; Zhao et al. 2013).

Os processos de separação de membranas estão sendo cada vez mais considerados como alternativas para o tratamento da água, uma vez que podem remover contaminantes particulados, incluindo protozoários e parasitas. No entanto, as membranas não podem efetivamente remover todos os contaminantes. Desta forma, a fim de melhorar o desempenho do tratamento, muitos processos de membrana foram acoplados de forma complementar a outros processos. Entre essas alternativas, a combinação de carvão ativado com processos de membrana tem recebido crescente consideração (Stoquart et al. 2012).

Geralmente, os processos de membrana são acionados por pressão e consomem muita energia, exigindo grandes vasos de pressão e altos custos (Voutchkov 2010). Outras desvantagens relacionadas à membrana no processo de tratamento de água são incrustações e entupimentos. A ocorrência desses fatores leva à redução na permeabilidade da membrana e a um consequente fluxo reduzido (Kim et al. 2009). 
Dessa forma, o objetivo do presente estudo foi avaliar a utilização de uma membrana de microfiltração polimérica em um módulo de membrana de microfiltração gravitacional e sua combinação com carvão ativado impregnado com prata e cobre, resultando em um processo híbrido para melhoria da qualidade da água para consumo humano.

\section{Metodologia}

\section{Impregnação de metais em carvão ativado}

Foi utilizado carvão ativado granular (CAG) obtido da casca de coco de dendê $(0,425$ a 1,18 mm, Bahiacarbon Agroindustrial Ltda). A técnica da impregnação úmida e as concentrações utilizadas foram baseadas em trabalhos anteriores (Bergamasco 2010; Yamaguchi et al. 2017). Em suma, uma mistura de 100 g CAG, $40 \mathrm{~mL}$ de água destilada, $30 \mathrm{~mL}$ de solução de sulfato de cobre e $30 \mathrm{~mL}$ de solução de nitrato de prata foram mantidos em rotaevaporador sob agitação de $20 \mathrm{rpm}$ à $60^{\circ} \mathrm{C}$ por 24 horas. Em seguida, o excesso de água foi evaporado em rotaevaporador à $80^{\circ} \mathrm{C}$, e a amostra foi seca em estufa à $100^{\circ} \mathrm{C}$ por 24 horas. Após a secagem, a amostra foi transferida para um cadinho de porcelana e submetida ao tratamento térmico em forno mufla à $350^{\circ} \mathrm{C}$ por 5 horas. Finalmente, a amostra foi submetida a uma lavagem com água destilada para remover partículas finas e metais não impregnados, seguida de nova secagem em estufa a $100^{\circ} \mathrm{C}$ por 24 horas.

\section{Caracterização dos meios filtrantes}

As morfologias superficiais dos meios filtrantes foram caracterizadas por meio de microscopia eletrônica de varredura (MEV) em microscópio Shimadzu SS-550 e microscopia eletrônica de transmissão (MET) em microscópio JEOL modelo JEM-1400. A composição das superfícies do CAG e o mapeamento elementar foram realizadas por análises de espectrometria de energia dispersiva (EDX) no mesmo equipamento de MEV. As estruturas cristalinas do CAG e CAG/AgCu foram identificadas por difração de raios-X (DRX) usando um modelo de Bruker AXS D8 Advance. A área de superfície, o volume de poros e o tamanho médio de poros de CAG e CAG/AgCu foram medidos em um sistema automático de sorção de gás Quantachrome Autosorb (Flórida, EUA) determinado por isotermas de adsorção-dessorção de N2 a $77 \mathrm{~K}$.

\section{Avaliação do módulo bíbrido de filtração}

O módulo híbrido de filtração gravitacional utilizado neste trabalho foi utilizado em trabalhos anteriores (Bergamasco et al. 2011; Silva et al. 2012; Yamaguchi et al. 2017). Em resumo, O CAG ou CAG/AgCu foi colocado no módulo de filtração, antes da membrana de microfiltração plana de acetato de celulose com diâmetro de poro de 3,0 $\mu \mathrm{m}$ (Advantec, Japão), em um filtro de leito fixo resultando em um sistema gravitacional híbrido. O filtro de leito fixo foi obtido adicionando-se $150 \mathrm{~g}$ de CAG ou CAG/ $\mathrm{AgCu}$ em um cartucho acrílico de $20 \mathrm{~cm}$ de altura. Testes adicionais foram realizados com o processo unitário, usando apenas a membrana, sem CAG ou CAG/ $\mathrm{AgCu}$. Todos os processos avaliados no sistema gravitacional são apresentados na Tabela 1. 
Tabela 1. Condições avaliadas neste estudo.

\begin{tabular}{ll}
\hline Abreviatura & Processo Avaliado \\
\hline $\mathrm{M}$ & Processo de membrana unitário \\
$\mathrm{M}+\mathrm{CAG}$ & Processo híbrido (membrana e CAG) \\
$\mathrm{M}+\mathrm{CAG} / \mathrm{AgCu}$ & Processo híbrido (membrana e CAG impregnado com prata e cobre) \\
\hline
\end{tabular}

Cada um dos três processos foi avaliado realizando cinco etapas com base em estudos anteriores (Bergamasco, et al., 2011, Silva, et al., 2012, Yamaguchi, et al., 2017): (1) determinação do fluxo permeado inicial com água deionizada com uma nova membrana, (2) filtração de água deionizada artificialmente contaminada com E. coli com uma concentração aproximada de $1 \times 10^{5} \mathrm{UFC} \mathrm{mL}^{-1}$ (3) quatro filtrações subsequentes de água da torneira de 120 min cada, (4) segunda filtração de água contaminada para determinar a remoção de E. coli após a filtração da água da torneira e (5) determinação do fluxo final do permeado com água deionizada.

\section{Análises de amostras de água}

Foram avaliadas as remoções de E. coli e cloro, turbidez, $\mathrm{pH}$ e fluxo de água filtrada para caracterizar a eficiência do módulo de filtração. Todos os procedimentos foram realizados com base no Standard methods for the examination of water and wastewater (APHA, et al., 2012). As características da água da torneira utilizada neste estudo estão listadas na Tabela 2.

Tabela 2. Características da água de torneira utilizada neste estudo.

\begin{tabular}{ll}
\hline Parâmetro & Valor \\
\hline Cor $(\mathrm{HU})$ & 0 \\
Turbidez $(\mathrm{NTU})$ & $0,09-1,21$ \\
Cloro livre $(\mathrm{mg} / \mathrm{L})$ & $0,02-0,63$ \\
$\mathrm{pH}$ & $6,86-7,51$ \\
\hline
\end{tabular}

\section{Resultados E Discussão}

\section{Caracterização dos meios filtrantes}

As micrografias de MEV e seu mapeamento elementar juntamente com o espectro de EDX encontram-se na Figura 1. 
Figura 1. Micrografia eletrônica de varredura para a amostra de membrana nova (a), CAG (b), e CAG/AgCu (c). Mapeamento elementar do CAG/AgCu para prata (d), cobre (e) e micrografia de eletrônica de transmissão para $\mathrm{CAG} / \mathrm{AgCu}(\mathrm{f})$.

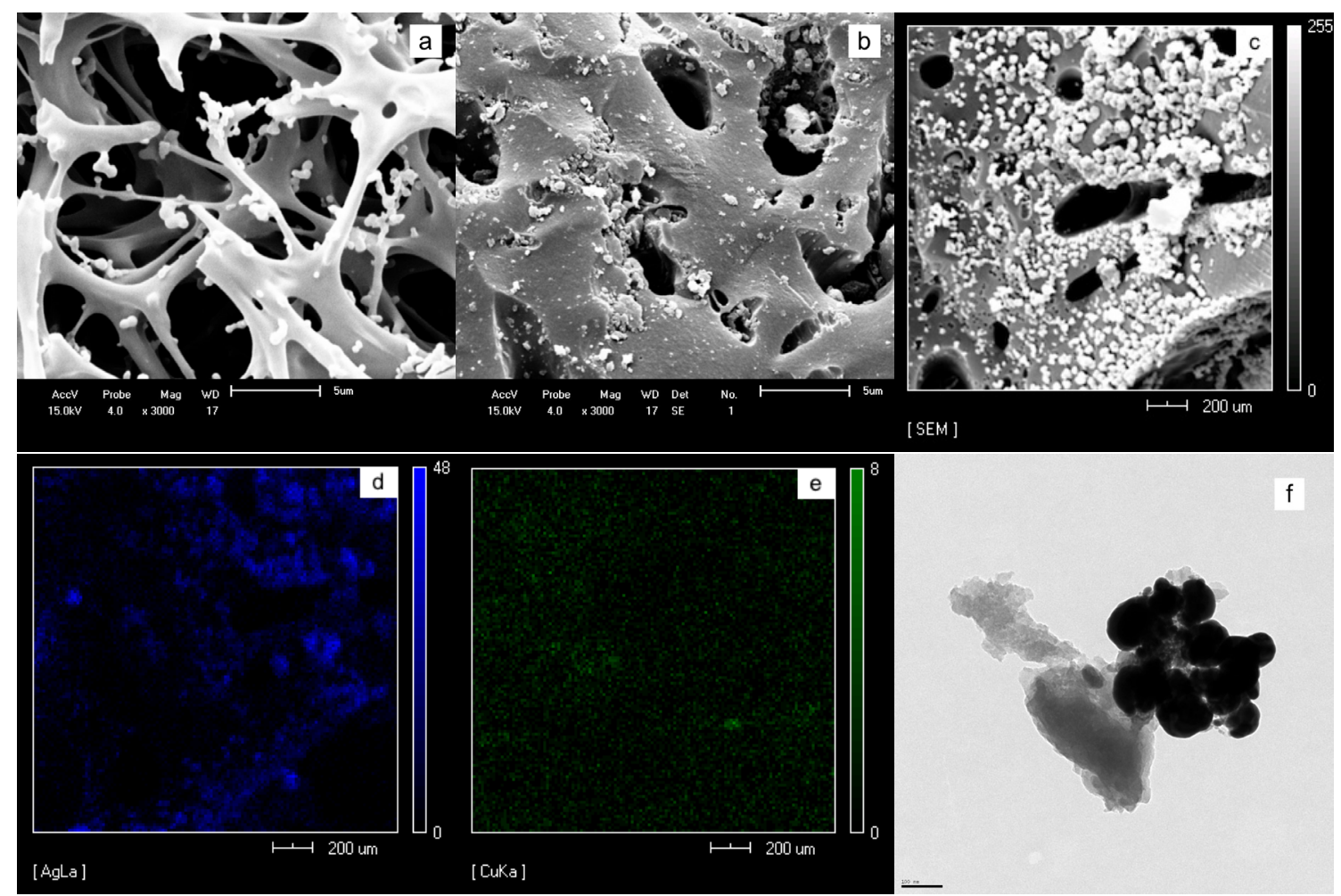

Os poros de aproximadamente $3 \mu \mathrm{m}$ foram observados na micrografia da membrana (Figura 1a) de acordo com o especificado pelo fabricante da membrana. A micrografia do CAG (Figura 1b) também apresentou macroporos amplos e uma morfologia plana, que pode ser verificado pelos resultados apresentados na Figura 1b, sendo uma estrutura típica de CAG a partir de casca de dendê, já que micrografias semelhantes foram relatadas por outros autores (Ngarmkam, et al., 2011, (Wong, Yoon, \& Jang, 2015). O CAG apresenta poros com vários tamanhos e formatos aleatórios, atribuindo-o uma alta capacidade de adsorção devido à presença de micro e mesoporos (Bansal \& Goyal, 2005). Comercialmente, o CAG é preparado a partir de recursos naturais, como casca de coco de dendê, gerando diferentes tamanhos e formas de poros durante os processos de carbonização e ativação, que vão de centenas de nanômetros a alguns micrômetros (Srinivasan, Shankar, \& Bandyopadhyaya, 2013).

De acordo com o mapeamento elementar do $\mathrm{CAG} / \mathrm{AgCu}$, notou-se que o cobre estava uniformemente disperso na superfície do CAG e a prata foi impregnada em forma de aglomerados de nanopartículas de prata (Figura 1c,d,e), sendo muito maiores que o tamanho médio dos poros do carvão ativado $(1,4-3,5 \mathrm{~nm}$, Tabela 3). Portanto, a fixação das microesferas de prata ocorreram na superfície e não dentro dos poros do CAG, e isto pode ser observado na análise de mapeamento elementar na cor azul da Figura 1d. Srinivasan (2013) encontrou micrografias de mapeamento elementar semelhantes aos obtidos neste trabalho para o CAG e prata. Esse resultado também pode ser confirmado pela análise de MET, cujas microesferas de aproximadamente $500 \mathrm{~nm}$ podem ser verificadas na Figura 1f, onde são observadas as nanopartículas de prata variando de 50 a $100 \mathrm{~nm}$ que formam estes aglomerados em forma de microesferas. Os resultados das análises de caracterização texturais do CAG estão apresentados na Tabela 3. 
Tabela 3. Características texturais do carvão ativado utilizado neste estudo.

\begin{tabular}{llllllll}
\hline \multirow{2}{*}{ Adsorvente } & \multicolumn{2}{l}{ Área $\left(\mathrm{m}^{2} / \mathrm{g}\right)$} & \multicolumn{2}{c}{ Volume $\left(\mathrm{cm}^{3} / \mathrm{g}\right)$} & \multicolumn{2}{c}{ Diâmetro $(\AA)$} \\
& BET & micro & meso & micro & meso & micro & meso \\
\hline $\mathrm{CAG}$ & 824 & 745 & 28 & 0,41 & 0,043 & 14 & 35 \\
$\mathrm{CAG} / \mathrm{AgCu}$ & 732 & 653 & 24 & 0,36 & 0,037 & 14 & 35 \\
\hline
\end{tabular}

O CAG apresentou características predominantemente microporosas de acordo com a análise de adsorçãodessorção de $\mathrm{N}_{2}$, com uma área de microporos de $745 \mathrm{~m}^{2} / \mathrm{g}$ correspondente a 90,4\% da área total específica e tamanho médio de microporos de $14 \AA$. Resultados similares foram obtidos para CAG de casca de dendê na literatura (Silva-Medeiros et al., 2016).

Comparando as áreas das amostras de CAG e CAG/AgCu, foi observada uma diminuição na área BET da amostra impregnada (-11\%), área de microporos (-12\%), mesoporos (-14\%), no volume de microporos $(-12 \%)$ e mesoporos (-14\%). Estes resultados sugerem que as nanopartículas de prata estão localizadas tanto nos mesoporos quanto em microporos (BandoszPetit, 2009) e indicam que a impregnação de prata e cobre leva ao bloqueio parcial de poros, como resultado da interação entre prata, cobre e a matriz de carbono (Goscianska, et al., 2012).

A Figura 2 apresenta os resultados da análise de DRX para o CAG e CAG/AgCu realizado para identificar as estruturas cristalinas impregnadas na sua superfície.

Figura 2. Difratogramas de DRX .

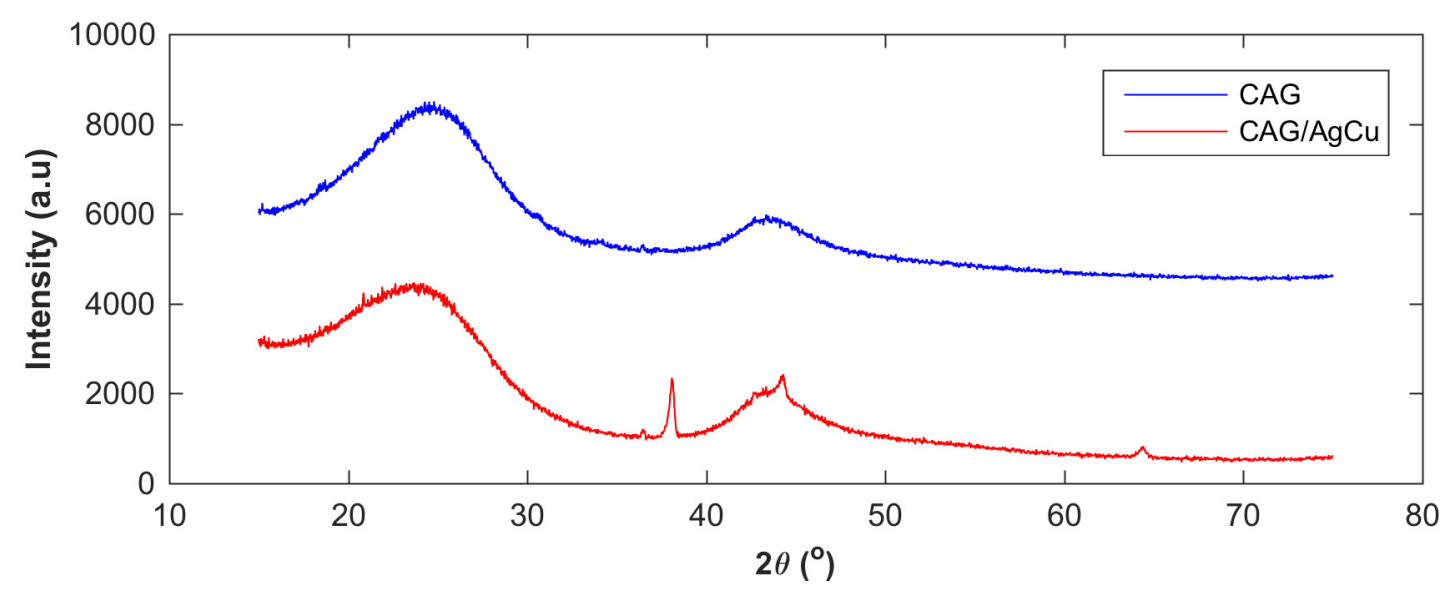

Nas análises de DRX, as fases cristalográficas do CAG e CAG/AgCu foram identificadas. Nos difratogramas de raios-X do CAG (Figura 2), os halos amplos sugerem a presença de uma fase amorfa do carbono. Os mesmos halos amorfos também foram identificados nos materiais carbonáceos estudados por Arakawa, Shimabuku-Biadola, de Lima Bazana, et al. (2019).

Nos difratogramas de raios-x obtidos do CAG/Ag, foram identificados planos relacionados aos compostos de prata em suas formas metálicas. Isso indica que o processo de impregnação do carvão ativado com os sais de nitrato de prata com subsequente decomposição térmica em atmosfera ambiente foi eficiente, pois foram encontrados cristalitos de tamanhos reduzidos, ou seja, nanopartículas de prata. Os picos localizados em $2 \theta$ próximos a $38^{\circ}$, $44^{\circ}$ e $64^{\circ}$ correspondem a reflexões de Bragg (111), (200) e (220), respectivamente, que podem ser indexados na estrutura cúbica da prata metálica (Baiocco, et al., 2016). Nenhum pico de cobre foi encontrado, sugerindo que o cobre impregnado é amorfo ou é muito pequeno para difratar, conforme justificado por KimYie (2005) e Muñiz et al. (2009) em outros trabalhos. 
A Figura 3 apresenta todos os parâmetros avaliados juntamente com a filtração da água da torneira no sistema de filtração, o fluxo inicial e final para todos os processos avaliados neste estudo.

Figura 3. Fluxo permeado de água da torneira para todos os processos avaliados neste estudo.
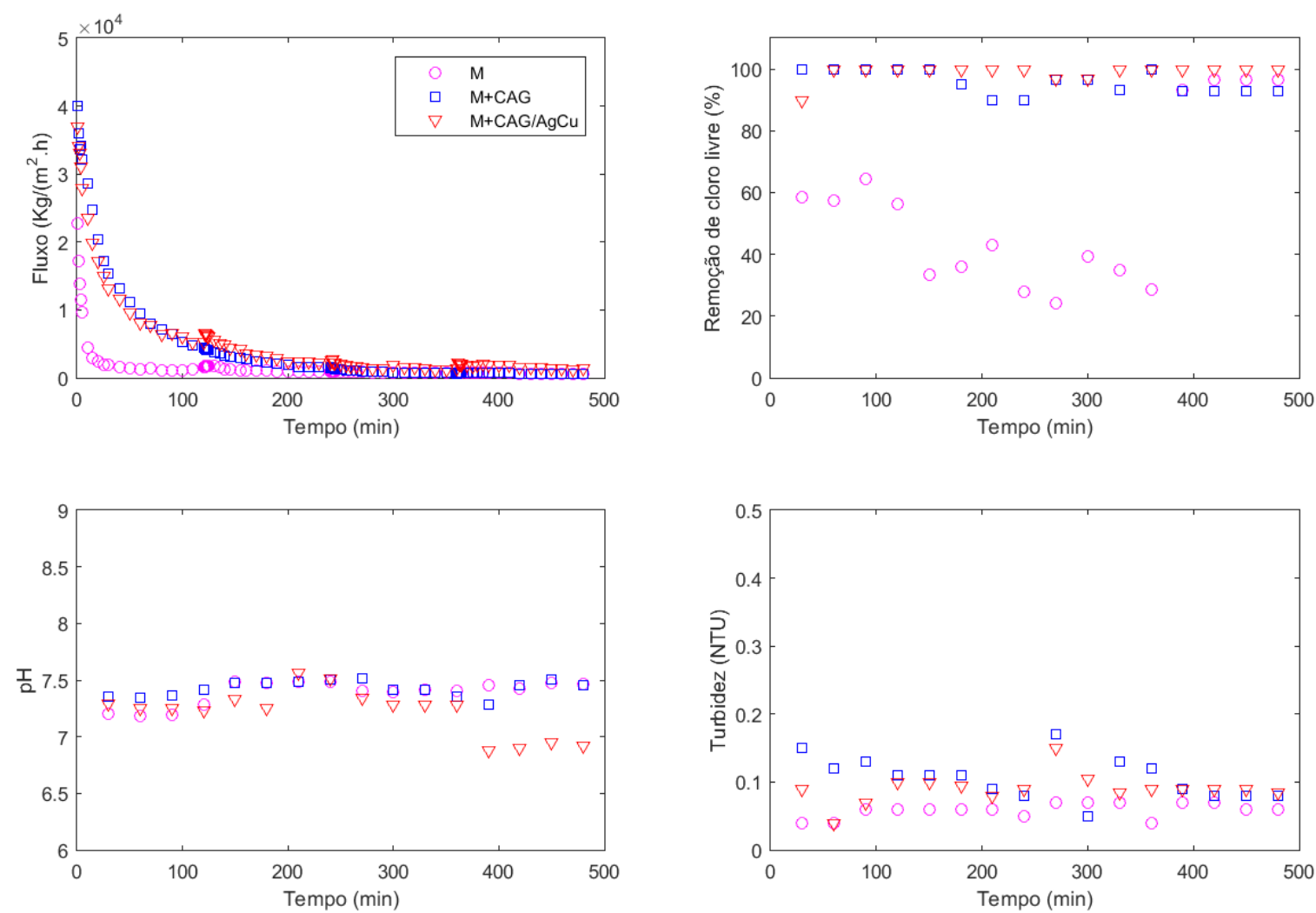

$\mathrm{Na}$ Figura 3 a foram observadas pequenas descontinuidades a cada 120 min no fluxo de permeado, uma vez que a filtração foi realizada em quatro etapas sequenciais de $120 \mathrm{~min} \mathrm{cada,} \mathrm{totalizando} 480 \mathrm{~min}$. Além disso, a Figura 3a revelou um fluxo mais alto nos sistemas híbridos, indicando que o CAG diminuiu a incrustação da membrana, garantindo um fluxo mais elevado. Isso pode ser justificado à adsorção de contaminantes no CAG, resultando na redução de contaminantes carregados na membrana (Kim, et al., 2009).

De acordo com a Figura 3c, a água filtrada apresentou um $\mathrm{pH}$ próximo à neutralidade, assim como a água da torneira (Tabela 2). Portanto, o sistema de filtração não alterou o pH da água. No entanto, a turbidez da água da torneira foi ligeiramente elevada quando utilizados os sistemas híbridos, porém em todos os casos, se mantiveram abaixo do exigido pela portaria 2914 (BRASIL, 2011). Isto possivelmente ocorreu devido às partículas finas de GAG solubilizadas no permeado.

O sistema de filtração híbrido apresentou um aumento significativo na remoção de cloro (Figura 3b), superiores a $90 \%$ em todas as amostras coletadas, enquanto o sistema de filtração para processo unitário (sem CAG) apresentou baixas remoções de cloro (24 - 97\%) confirmando que a microfiltração sozinha não remove alguns contaminantes da água e que o CAG pode ser usado como uma tecnologia combinada para melhorar a qualidade da água (Kim, et al., 2009). Os resultados de avaliação antibacteriana estão apresentados na Tabela 4. 
Tabela 4. Resultados de avaliação antibacteriana.

\begin{tabular}{lcc}
\hline Configuração & $\begin{array}{c}\text { Remoção de E. coli }(\log ) \\
\text { Inicial }\end{array}$ & Final \\
\hline $\mathrm{M}$ & 0,5 & 5,4 \\
$\mathrm{M}+\mathrm{CAG}$ & 0,5 & 3,3 \\
$\mathrm{M}+\mathrm{CAG} / \mathrm{AgCu}$ & 3,7 & 5,1 \\
\hline
\end{tabular}

Em relação à remoção final de E. coli apresentada na Tabela 4, todos os sistemas de filtração obtiveram remoções finais relevantes de E. coli devido à incrustação da membrana após a filtração da água da torneira. A remoção inicial de E. coli, no entanto, foi 7,4 vezes maior no sistema de filtração híbrida com GAG/AgCu quando comparado com os outros sistemas de filtração. De acordo com outros autores as nanopartículas dispersas na superfície do CAG podem ser mais acessíveis às bactérias melhorando a atividade antibacteriana do material (Ruparelia, et al., 2008, Wang, et al., 2015, Ilnicka, et al., 2016). Segundo Srinivasan et al. (2013) as nanopartículas têm a capacidade de atravessar a membrana celular e restringir significativamente o crescimento de bactérias.

As bactérias apresentam maior sensibilidade ao material poroso modificado com o GAC/AgCu, Outros autores também estudaram e comprovaram o efeito inibitório dessas nanopartículas sobre bactérias. Na avaliação de materiais modificados sem a combinação de nanopartíclas de prata e cobre, ou seja, o material modificado apenas com nanopartículas de cobre ou prata separadamente (Arakawa, Shimabuku-Biadola, de Lima Bazana, et al., 2019; Yamaguchi, Abe, Medeiros, Vieira, \& Bergamasco, 2017), foi possível verificar que ocorre um efeito sinérgico das nanopartículas de prata e cobre impregnados no carvão ativado, potencializando o seu efeito antibacteriano.

\section{CONCLUSÕES}

O método de impregnação de prata e cobre garantiu ao GAC/AgCu maior remoção de cloro, maiores fluxos iniciais de permeado, não alterou o pH e não aumentou significativamente a turbidez. O CAG/AgCu também melhorou a eficiência bactericida inicial em 7,4 vezes em relação ao CAG. Portanto, os resultados obtidos mostraram que é possível melhorar a qualidade da água da torneira em um sistema híbrido de membrana gravitacional e carvão ativado impregnado com prata e cobre.

\section{REFERÊNCIAS}

APHA, AWWA, WEF. 2012. Standard Methods for the Examination of Water \& Wastewater, 22 ed edn: American Public Health Association, 1496 p.

Brick T, Primrose B, Chandrasekhar R, Roy S, Muliyil J, Kang G. 2004. Water contamination in urban south India: household storage practices and their implications for water safety and enteric infections. International journal of hygiene and environmental health, 207(5):473-480.

Peter-Varbanets M, Zurbrügg C, Swartz C, Pronk W. 2009. Decentralized systems for potable water and the potential of membrane technology. Water Research, 43(2):245-265.

Silva FV, Yamaguchi NU, Lovato GA, Silva FA, Reis MH, Amorim MT, Tavares CR, Bergamasco R. 2012. Effects of coconut granular activated carbon pretreatment on membrane filtration in a gravitational driven process to improve drinking water quality. Environmental technology, 33(4-6):711-716. 
Bhatnagar A, Hogland W, Marques M, Sillanpää M. 2013. An overview of the modification methods of activated carbon for its water treatment applications. Chemical Engineering Journal, 219(0):499-511.

Zhao Y, Wang Z-q, Zhao X, Li W, Liu S-x. 2013. Antibacterial action of silver-doped activated carbon prepared by vacuum impregnation. Applied Surface Science, 266(0):67-72.

Stoquart C, Servais P, Bérubé PR, Barbeau B. 2012. Hybrid Membrane Processes using activated carbon treatment for drinking water: A review. Journal of Membrane Science, 411-412:1-12.

Voutchkov N. 2010. Considerations for selection of seawater filtration pretreatment system. Desalination, 261(3):354-364.

Kim K-Y, Kim H-S, Kim J, Nam J-W, Kim J-M, Son S. 2009. A hybrid microfiltration-granular activated carbon system for water purification and wastewater reclamation/reuse. Desalination, 243(1-3):132-144.

Bergamasco R: Carvão ativado impregnado com prata e cobre para eliminação de microrganismos da água. In. Brasil: Universidade Estadual de Maringá; 2010.

Yamaguchi NU, Abe SAL, Medeiros FVdS, Vieira AMS, Bergamasco R. 2017. Hybrid gravitational microfiltration system for drinking water purification. Revista Ambiente \& Água, 12:168-178.

Bergamasco R, da Silva FV, Arakawa FS, Yamaguchi NU, Reis MHM, Tavares CJ, de Amorim MTPS, Tavares CRG. 2011. Drinking water treatment in a gravimetric flow system with TiO2 coated membranes. Chemical Engineering Journal, 174(1):102-109.

Ngarmkam W, Sirisathitkul C, Phalakornkule C. 2011. Magnetic composite prepared from palm shell-based carbon and application for recovery of residual oil from POME. J Environ Manage, 92(3):472-479.

Srinivasan NR, Shankar PA, Bandyopadhyaya R. 2013. Plasma treated activated carbon impregnated with silver nanoparticles for improved antibacterial effect in water disinfection. Carbon, 57(0):1-10.

Baiocco D, Lavecchia R, Natali S, Zuorro A. 2016. Production of Metal Nanoparticles by Agro-Industrial Wastes: A Green Opportunity for Nanotechnology. Chemical Engineering Transactions, 47:6.

Kim DJ, Yie JE. 2005. Role of copper chloride on the surface of activated carbon in adsorption of methyl mercaptan. Journal of Colloid and Interface Science, 283(2):311-315.

Muñiz G, Fierro V, Celzard A, Furdin G, Gonzalez-Sánchez G, Ballinas ML. 2009. Synthesis, characterization and performance in arsenic removal of iron-doped activated carbons prepared by impregnation with $\mathrm{Fe}(\mathrm{III})$ and $\mathrm{Fe}$ (II). Journal of Hazardous Materials, 165(1-3):893-902.

Bandosz TJ, Petit C. 2009. On the reactive adsorption of ammonia on activated carbons modified by impregnation with inorganic compounds. Journal of Colloid and Interface Science, 338(2):329-345.

Goscianska J, Nowak I, Nowicki P, Pietrzak R. 2012. The influence of silver on the physicochemical and catalytic properties of activated carbons. Chemical Engineering Journal, 189-190(0):422-430.

BRASIL: Portaria $\mathbf{n}^{\circ}$ 2.914, de 12 de dezembro de 2011. Dispõe sobre os procedimentos de controle e de vigilância da qualidade da água para consumo humano e seu padrão de potabilidade. In.: Ministério da Saúde; 2011. 
Wang W, Xiao K, He T, Zhu L. 2015. Synthesis and characterization of Ag nanoparticles decorated mesoporous sintered activated carbon with antibacterial and adsorptive properties. Journal of Alloys and Compounds, 647:1007-1012.

Ilnicka A, Walczyk M, Lukaszewicz JP, Janczak K, Malinowski R. 2016. Antimicrobial carbon materials incorporating copper nano-crystallites and their PLA composites. Journal of Applied Polymer Science, 133(20):n/a-n/a.

Ruparelia JP, Chatterjee AK, Duttagupta SP, Mukherji S. 2008. Strain specificity in antimicrobial activity of silver and copper nanoparticles. Acta biomaterialia, 4(3):707-716.

Arakawa, F. S., Shimabuku-Biadola, Q. L., de Lima Bazana, S., Silva, M. F., de Abreu Filho, B. A., \& Bergamasco, R. (2019). Activated carbon impregnation with ag and cu composed nanoparticles for escherichia coli contaminated water treatment. The Canadian Journal of Chemical Engineering, 97(9), 2408-2418. doi:10.1002/cjce.23471

Arakawa, F. S., Shimabuku-Biadola, Q. L., Fernandes Silva, M., \& Bergamasco, R. (2019). Development of a new vacuum impregnation method at room atmosphere to produce silver-copper oxide nanoparticles on activated carbon for antibacterial applications. Environ Technol, 1-12. doi:10.1080/09593330.2019.1567607

Bansal, R. C., \& Goyal, M. (2005). Boca Raton, Florida: Taylor \& Francis Group.

Montgomery, M. A., \& Elimelech, M. (2007). Water And Sanitation in Developing Countries: Including Health in the Equation. Environmental Science \& Technology, 41(1), 17-24. doi:10.1021/es072435t

Silva-Medeiros, F. V., Consolin-Filho, N., Xavier de Lima, M., Bazzo, F. P., Barros, M. A. S. D., Bergamasco, R., \& Tavares, C. R. G. (2016). Kinetics and thermodynamics studies of silver ions adsorption onto coconut shell activated carbon. Environ Technol, 1-7. doi:10.1080/09593330.2016.1176076

Srinivasan, N. R., Shankar, P. A., \& Bandyopadhyaya, R. (2013). Plasma treated activated carbon impregnated with silver nanoparticles for improved antibacterial effect in water disinfection. Carbon, 57(0), 1-10. doi:http://dx.doi.org/10.1016/j. carbon.2013.01.008

Wong, K. T., Yoon, Y., \& Jang, M. (2015). Enhanced recyclable magnetized Palm shell waste-based powdered activated carbon for the removal of ibuprofen: insights for kinetics and mechanisms. PLoS One, 10(10), e0141013.

Yamaguchi, N. U., Abe, S. A. L., Medeiros, F. V. d. S., Vieira, A. M. S., \& Bergamasco, R. (2017). Hybrid gravitational microfiltration system for drinking water purification. Revista Ambiente \& Água, 12, 168-178. 